\title{
Effect of Protocol of Care of Patients Undergoing Urinary Catheterization on Nurses' Knowledge
}

\author{
Prof. Labiba Abd Elkader Mohamed, Prof. Dr. Mamdouh Mohamad AIMezaien , \\ Ass. Prof. Amal Bakr Abo El-Ata, and Amira Tag Mohamed Abo-El Nasser \\ Prof of Medical Surgical Nursing ， Faculty of Nursing, Cairo University, \\ Professor of vascular surgery, Faculty of Medicine, Suez Canal University, Assistant \\ Professor of Medical - Surgical Nursing, Faculty of Nursing - Port Said University, \\ Clinical Instructor, Al-Azhar University, Egypt.
}

\begin{abstract}
Background: The indwelling urinary catheter (IUC) is a widely utilized device in modern hospital environments, yet they are not always used appropriately in hospital settings and can result in prolonged use and improper management, increasing risk of infections and length of stay. Hence, professional must have knowledge related to the management of urinary catheterization. Aim of the study was to evaluate the impact of protocol of care of patients undergoing urinary catheterization on nurses' knowledge. Subjects and Method: A quasi- experimental research design was used to conduct this study. The study was carried out at Intensive Care Unit, Urology Department and Department of Internal Medicine at Al-Azhar University hospital in Damietta City to test the hypotheses that positive changes in nurses' knowledge after implementing the protocol of care for patients undergoing urinary catheterization. A convenient sample of 50 nurses was included. One tool was used for data collections, part (1) Structured Interview Questionnaire tool that includes demographic data part (2) Nurse's Knowledge Assessment tool, to assess nurse's Knowledge about urinary catheterization and urinary catheter care Results: demonstrated that there were statistically significant improvements in the total score of nurses' knowledge regarding care of patients undergoing urinary catheterization throughout the protocol intervention . Recommendations: The study recommended that providing ongoing in-service education for nurses to update their knowledge related to care of patients undergoing urinary catheterization and catheter-associated problems.
\end{abstract}

Key words: Protocol of Care, Urinary catheterization 


\section{INTRODUCTION}

Urinary catheterization is a routine medical procedure that allows direct drainage of the urinary bladder into an attached bag. It consists in the insertion of a catheter into a patient's bladder. The urinary catheterization is used to drain urine by aseptic technique and painless insertion of a catheter (tube) into a patient's bladder for withdrawing urine (Essomba et al., 2013 and Savage, 2015).

Urinary catheter considered one of the most invasive medical devices used in the acute care setting which involves introducing hollow tube through urethra into the bladder. Urinary catheters are a necessity for a substantial percentage of the population, including hospitalized patients, residents in long-term care institutions, and those with various urological or genitourinary disorders (Perry \& Portter, 2010 and Ignatavicicius \& Workman, 2013).

Indwelling urinary catheterization has a number of indications such as accurately monitor the urinary output of critically ill patients, increase the comfort of terminally or severely ill patients also catheterization helps to manage skin damage caused by incontinence, when all other methods of managing urinary incontinence have failed and use for maintaining a continuous outflow of urine for patients undergoing surgical procedures, as part of standard preoperative preparation, maintaining a continuous outflow of urine for patient with voiding difficulties because of neurological disorders that cause paralysis or loss of sensation affecting urination, and providing immediate treatment of acute urinary retention (Fakih et al., 2011and Tiwari et al., 2012).

The biggest risk factor for acquiring urinary tract infection (UTI) is the presence of an indwelling urinary catheter (Weber et al., 2011). Each day an indwelling catheter is in place increases the risk of infection an estimated 3\% to $7 \%$.Nurse driven protocols are effective in catheter associated urinary tract infection (CAUTI) reduction, thus improving quality of care for patients in the hospital setting. Nurse driven protocols are beneficial over physician reminder systems because there is less delay in removal of the catheter. Nurses are at the forefront of care and require education, empowerment and support for a nurse driven protocol to be most effective (Lo et al., 2014 and Fisher, 2015). 
There is a body of up-to-date research evidence relating to the appropriate management of patients with indwelling urinary catheters (IUC). Healthcare workers are in a position to reduce the morbidity and mortality related to the use of urinary catheters.

\section{AIM OF STUDY:}

The aim of this study was to; evaluate the impact of protocol of care of patients undergoing urinary catheterization on nurses' knowledge.

\section{Research Hypotheses:}

To fulfill the aim of this study the following hypothesis was formulated:

- There will be positive changes in nurses' knowledge after implementing the protocol of care for patients undergoing urinary catheterization.

\section{SUBJECT AND METHOD:}

\section{Research Design:}

A quasi-experimental study design was used for the conduction of this study.

\section{Setting:}

This study was conducted at Intensive Care Unit, Urology Department and Department of Internal Medicine at Al-Azhar University hospital in Damietta city.

\section{Subjects:}

A convenient sample of 50 nurses working with patients undergoing urinary catheterization at Intensive Care Unit, Urology Department and Department of Internal Medicine, at Al-Azhar University hospital in Damietta city.

\section{Exclusion criteria:}

- Student nurses of any educational level.

- Nurses who refuse to participate in the study

\section{Tools of data collection:}

Tools used in this study were: one tool were used in this study

\section{Part (1): Structured interview Questionnaire:}

It was developed by the researcher based on the review of recent related literature to assess the nurses' knowledge regarding nursing care provided to patients undergoing 
urinary catheterization .It Included items related to socio- demographic characteristics and work related data of the studied nurses such as age, gender, marital status, working unit, level of education, years of experience and attending training program related to urinary catheter.

Part (II): Nurses' knowledge assessment: It included a group of questions to assess the nurse's knowledge in relation to key components of urinary catheterization and catheter care. Questions related to Infection control measures (12 MCQ questions), questions related to Precautions during urinary catheter insertion (10 MCQ questions) and questions related to nursing care for urinary catheter (16 MCQ questions).

\section{Scoring system:}

\section{Scoring system:}

Regarding the scoring system for nurse's knowledge, all knowledge variables were weighted according to the item included in the answer of each question. All questions marks was summed and divided by the number of questions to obtain the mean knowledge of each nurse for each part and as a total. Knowledge below 75\% was considered unsatisfactory while those equal to or above $75 \%$ was considered satisfactory.

\section{Operational Design:}

The operational design of this study included preparatory phase, content validity, pilot study, and field work.

\section{Preparatory Phase:}

It included reviews of current and recent local and international related literatures, and theoretical knowledge of various aspects of the study using books, articles, and internet periodicals and magazines in order to develop the data collection tools.

\section{Content Validity:}

It was ascertained by a Jury consisting of seven experts of professors and lecturers from the medical surgical department; Faculty of nursing and from medicine, surgery and urology department Faculty of Medicine, Al-Azhar University who revised the tools for clarity, relevance, comprehensiveness, understanding and ease for implementation, according to their opinion modifications were applied. 


\section{Pilot study:}

Pilot study had been undertaken before starting the data collection phase. It was carried out on $10 \%$ of participants to test the feasibility and applicability of the tool to estimate the time needed to complete the tool. According to the pilot study necessary modifications were done. The subjects included in the pilot study were excluded from the study sample.

\section{Field work description}

Field study was conducted from the beginning of May (2014) to the end of May (2015). The study was carried out through the following phases:

\section{Assessment phase:}

In this phase after finalization of the tools, the researcher assessed nurses' learning needs using (part II of the tool). The Tool was designed to assess nurses' knowledge related to providing care for patients undergoing urinary catheterization. The researcher introduced this tool to each nurse and asked him/her to fill it out. The time taken to fill the tool was from 30 minutes to 60 minutes. Moreover, the researcher assessed available place, time, equipment, supplies, and instructional materials for conduction of the protocol of care.

\section{The protocol of care development phase}

The protocol of care was developed based on the identified needs and demands of nurses gathered in assessment phase and review of related literature. This phase included the following;

\section{A. Setting objectives:}

The aim of protocol was to improve nurses performance related to care of patients undergoing urinary catheterization though:

$>$ Improve nurses' knowledge related to care of patients undergoing urinary catheterization

\section{B. Preparation of the content:}

Content covered all areas about caring of patients undergoing urinary catheterization 
was prepared which included the following: infection control related urinary catheter, contraindications of urinary catheter, preparation during urinary catheter insertion, and nursing care for urinary catheter

\section{Planning of action:}

In this phase, the researcher designed a plan for a protocol of care implementation

\section{Implementation phase:}

After official permission was taken from the concerned study setting. The researcher took the list of nurses who met the inclusion criteria. The participated nurses were divided into 10 groups, each consisted of five nurses. Each group was attended at conference room separately during morning and afternoon shift. The purpose and aim of the study was explained, then the researcher collects data about demographic characteristics using tool (part I). This session is considered as introductory session.

At the beginning of each session, pretest related to the session content was provided to participants, followed by hands out. During the session, the researcher teach content in a clear, simple language using lectures, illustrative pictures and discussion giving feedback using positive verbal words.

At the end of each session the researcher, close the session by summary for the main points. Posttest was at the end of the $4^{\text {th }}$ session using tool (part II).

\section{Evaluation phase:}

The protocol of care was evaluated three times using the tool used to evaluate the studied nurses .Evaluation was done three times, first time: immediately after protocol implementation, second time after three months, and third time: after six month.

\section{Ethical Consideration:}

Explain the aim of the study to the directors of Intensive Care Unit, Department of Urology and Internal Medicine to take their permission to start this study .Oral consent was taken from the study subjects after explaining the aims and nature of the study to them, and they were assured that the information collected would be treated confidentially and used for the research purpose only, and they have the right to withdraw from the study at any time. 


\section{Statistical Design:}

The collected data organized, tabulated and statistically analyzed using statistical package for social science (SPSS) version 16 for windows, running on IBM compatible computer. Qualitative data (categorical data) were expressed as relative frequency (number) and percent distribution, and for comparison between groups, the Chi square $\left(\mathrm{X}^{2}\right)$ or Mann-Whitney test $(\mathrm{Z})$ was calculated. Quantitative data were expressed as mean $\pm \mathrm{SD}$, and for comparison between two means, the student $(\mathrm{t})$ test was calculated. For interpretation of results, the $p$ value $\leq 0.05$ was considered significant.

\section{RESULTS:}

Table (1) : shows the demographic and work related data of studied nurses. It revealed that (96\%) of the studied nurses were females and (90\%) their age from 21 to 36 years. There were $46 \%$ of studied nurses had technical nursing institute diploma, while only (16\%) had nursing bachelor; and (48\%) had less than five years of experience and $(50.0 \%)$ working in intensive care unit (ICU) and (30\%) work in urology unit. All studied nurses (100\%) have not any previous training courses about urinary catheterization.

Table (2) :Shows that there were high statistical significant differences in knowledge scores related to all items of infection control policies during the urinary catheterization throughout the protocol intervention among studied nurses $(\mathrm{p}<0.001)$. There was $(28 \%)$ before intervention,(100\%) immediately after,(98\%) 3months after and (96\%) 6 months after protocol intervention among studied nurses have satisfactory score knowledge related to infection control policies during the urinary catheterization. There was high statistical significant difference $\left(X^{2}=117.31, p<0.001\right)$ in total knowledge score among studied nurses throughout intervention duration

Table (3): Denotes that before intervention, (2\%) have satisfactory knowledge score, immediate after (94\%), 3 month after (84\%) and 6 month after intervention (72\%) have total satisfactory knowledge score. Moreover, there were statistically significant differences $\left(\mathrm{X}^{2}=111.62, \mathrm{p}<0.001\right)$ about nursing care for patients undergoing urinary catheterization while, there was no statistical significant difference $\left(X^{2}=3.72 \mathrm{p}=0.27\right)$ among studied nurses in knowledge score about patient ability to urinate after urinary catheter removal. 
Table (4) : reveals that there were statically significant difference in the total score of nurses' knowledge regarding urinary catheter care throughout the protocol intervention between posttest, pre protocol first follow up and pre, second follow up, and pre protocol $\left(\mathrm{X}^{2}=146.2 \mathrm{P}<0.001\right)$. A high statistical significant improvement in nurse's knowledge were found between the immediate posttest and the pre protocol level, between pre protocol and 3 month after $(\mathrm{P}<0.001)$. There were no statistically significant differences between immediately posttest and first follow up $(\mathrm{P}=0.56)$ and between first and second follow up $(\mathrm{p}=0.31)$. In addition, there was no statistical significant difference between first and second follow-up $(\mathrm{P}=0.64)$.

Table (5): Shows that there were high statistically significant differences between the knowledge of studied nurses and their education throughout the protocol intervention $(\mathrm{p}=0.001)$ except in immediately after protocol No statistically significant difference was found $(\mathrm{p}=0.09)$.

Table (1): Distribution of the studied nurses according to their demographic characteristics and work related data $\left(\mathrm{No}_{\mathrm{O}}=50\right)$

\begin{tabular}{|c|c|c|c|}
\hline \multicolumn{2}{|l|}{ Items } & $\mathbf{N}$ & $\%$ \\
\hline \multirow[t]{2}{*}{-Age } & Less than 21years & 5 & 10.0 \\
\hline & 21 to 36 years & 45 & 90.0 \\
\hline \multirow[t]{2}{*}{-Gender } & Male & 2 & 4.0 \\
\hline & Female & 48 & 96.0 \\
\hline \multirow[t]{4}{*}{-Marital state } & Single & 9 & 18.0 \\
\hline & Married & 40 & 80.0 \\
\hline & Divorced & 1 & 2.0 \\
\hline & Widow & 0 & 0.0 \\
\hline \multirow[t]{3}{*}{-Education level } & Secondary school diploma & 19 & 38.0 \\
\hline & Technical nursing institute diploma & 23 & 46.0 \\
\hline & Bch degree of nursing & 8 & 16.0 \\
\hline \multirow[t]{3}{*}{-Years of experience } & Less than 5 years & 24 & 48.0 \\
\hline & 5 to 10 years & 20 & 40.0 \\
\hline & More than 10 years & 6 & 12.0 \\
\hline \multirow[t]{3}{*}{-Work Department } & Urology & 15 & 30.0 \\
\hline & ICU & 25 & 50.0 \\
\hline & Internal medicine & 10 & 20.0 \\
\hline \multirow[t]{2}{*}{-Training course } & Yes & 0 & 0.0 \\
\hline & No & 50 & 100.0 \\
\hline
\end{tabular}


Table (2): Percentage distribution of studied nurses' satisfactory knowledge regarding the infection control policies during the urinary catheterization throughout the protocol intervention $(\mathrm{No}=50)$

\begin{tabular}{|c|c|c|c|c|c|c|c|c|c|c|}
\hline \multirow[t]{2}{*}{ Items } & \multicolumn{2}{|c|}{$\begin{array}{l}\text { Before } \\
\text { intervention }\end{array}$} & \multicolumn{2}{|c|}{$\begin{array}{c}\text { Immediately } \\
\text { After }\end{array}$} & \multicolumn{2}{|c|}{3 Months After } & \multicolumn{2}{|c|}{6 Months After } & \multirow[t]{2}{*}{ 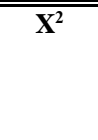 } & \multirow[t]{2}{*}{ P-value } \\
\hline & $\mathbf{N}$ & $\%$ & $\mathbf{N}$ & $\%$ & $\mathbf{N}$ & $\%$ & $\mathbf{N}$ & $\%$ & & \\
\hline $\begin{array}{l}\text { The best Effective method to } \\
\text { prevent infection }\end{array}$ & $\begin{array}{l}4 \\
4\end{array}$ & $\begin{array}{c}88.0 \\
\%\end{array}$ & 50 & $100.0 \%$ & 50 & $\begin{array}{c}100.0 \\
\%\end{array}$ & 50 & $100.0 \%$ & 18.55 & $<0.001 * *$ \\
\hline $\begin{array}{l}\text { Hand washing before catheter } \\
\text { insertion }\end{array}$ & $\begin{array}{l}1 \\
8\end{array}$ & $\begin{array}{c}36.0 \\
\%\end{array}$ & 49 & $98.0 \%$ & 45 & $90.0 \%$ & 45 & $90.0 \%$ & 72.61 & $<0.001 * *$ \\
\hline $\begin{array}{l}\text { Hand washing before urine bag } \\
\text { emptying }\end{array}$ & $\begin{array}{l}1 \\
0\end{array}$ & $\begin{array}{c}20.0 \\
\%\end{array}$ & 47 & $94.0 \%$ & 46 & $92.0 \%$ & 45 & $90.0 \%$ & 101.24 & $<0.001^{* *}$ \\
\hline $\begin{array}{l}\text { Hand washing before taking } \\
\text { urine sampling }\end{array}$ & $\begin{array}{l}1 \\
2\end{array}$ & $\begin{array}{c}24.0 \\
\%\end{array}$ & 47 & $94.0 \%$ & 43 & $86.0 \%$ & 41 & $82.0 \%$ & 75.64 & $<0.001 * *$ \\
\hline Site of catheter insertion & $\begin{array}{l}4 \\
1\end{array}$ & $\begin{array}{c}82.0 \\
\%\end{array}$ & 50 & $100.0 \%$ & 49 & $98.0 \%$ & 49 & $98.0 \%$ & 20.29 & $<0.001^{* *}$ \\
\hline Follow Sterilization basics & $\begin{array}{l}3 \\
0\end{array}$ & $\begin{array}{c}60.0 \\
\%\end{array}$ & 46 & $92.0 \%$ & 46 & $92.0 \%$ & 46 & $92.0 \%$ & 28.57 & $<0.001 * *$ \\
\hline $\begin{array}{l}\text { During catheter insertion use } \\
\text { gloves }\end{array}$ & $\begin{array}{l}3 \\
7\end{array}$ & $\begin{array}{c}74.0 \\
\%\end{array}$ & 50 & $100.0 \%$ & 49 & $98.0 \%$ & 49 & $98.0 \%$ & 33.08 & $<0.001^{* *}$ \\
\hline Urethral opening disinfection & $\begin{array}{l}4 \\
2\end{array}$ & $\begin{array}{c}84.0 \\
\%\end{array}$ & 50 & $100.0 \%$ & 49 & $98.0 \%$ & 49 & $98.0 \%$ & 17.26 & $0.001 * *$ \\
\hline $\begin{array}{llll}\text { Change glove } & \text { before } & \text { bag } \\
\text { emptying } & & & \\
\end{array}$ & $\begin{array}{l}3 \\
8\end{array}$ & $\begin{array}{c}76.0 \\
\%\end{array}$ & 49 & $98.0 \%$ & 49 & $98.0 \%$ & 49 & $98.0 \%$ & 26.16 & $<0.001 * *$ \\
\hline $\begin{array}{l}\text { Daily cleaning around urinary } \\
\text { catheter }\end{array}$ & $\begin{array}{l}1 \\
3\end{array}$ & $\begin{array}{c}26.0 \\
\%\end{array}$ & 45 & $90.0 \%$ & 43 & $86.0 \%$ & 43 & $86.0 \%$ & 70.23 & $<0.001 * *$ \\
\hline $\begin{array}{lll}\text { Following } & \text { Infections } & \text { control } \\
\text { policies } & & \\
\end{array}$ & $\begin{array}{l}3 \\
6\end{array}$ & $\begin{array}{c}72.0 \\
\%\end{array}$ & 50 & $100.0 \%$ & 45 & $90.0 \%$ & 44 & $88.0 \%$ & 18.42 & $<0.001 * *$ \\
\hline Disinfection urethral opening & $\begin{array}{l}3 \\
7\end{array}$ & $\begin{array}{c}74.0 \\
\%\end{array}$ & 50 & $100.0 \%$ & 48 & $96.0 \%$ & 47 & $94.0 \%$ & 24.66 & $<0.001^{* *}$ \\
\hline Total & $\begin{array}{l}1 \\
4\end{array}$ & $\begin{array}{c}28.0 \\
\%\end{array}$ & 50 & $100.0 \%$ & 49 & $98.0 \%$ & 48 & $96.0 \%$ & 117.31 & $<0.001 * *$ \\
\hline
\end{tabular}


Table (3) :Percentage distribution of studied nurses' satisfactory knowledge regarding nursing care for patients undergoing urinary catheterization throughout the protocol intervention $(\mathrm{No}=50)$

\begin{tabular}{|c|c|c|c|c|c|c|c|c|c|c|}
\hline \multirow[t]{2}{*}{ Items } & \multicolumn{2}{|c|}{$\begin{array}{l}\text { Before } \\
\text { intervention }\end{array}$} & \multicolumn{2}{|c|}{$\begin{array}{c}\text { Immediately } \\
\text { After }\end{array}$} & \multicolumn{2}{|c|}{3 months After } & \multicolumn{2}{|c|}{6 months after } & \multirow[t]{2}{*}{$\mathrm{X}^{2}$} & \multirow[t]{2}{*}{ P-value } \\
\hline & $\mathbf{N}$ & $\%$ & $\mathbf{N}$ & $\%$ & $\mathbf{N}$ & $\%$ & $\mathbf{N}$ & $\%$ & & \\
\hline Fluids intake per day & 27 & $54.0 \%$ & 45 & $90.0 \%$ & 43 & $\begin{array}{c}86.0 \\
\%\end{array}$ & 43 & $86.0 \%$ & 25.43 & $<0.001^{* *}$ \\
\hline Observe input output fluids & 10 & $20.0 \%$ & 45 & $90.0 \%$ & 41 & $\begin{array}{c}82.0 \\
\%\end{array}$ & 37 & $74.0 \%$ & 67.57 & $<0.001 * *$ \\
\hline Output fluids per day & 22 & $44.0 \%$ & 44 & $88.0 \%$ & 37 & $\begin{array}{c}74.0 \\
\%\end{array}$ & 37 & $74.0 \%$ & 24.57 & $<0.001 * *$ \\
\hline To keep urine flow ... & 18 & $36.0 \%$ & 46 & $92.0 \%$ & 40 & $\begin{array}{c}80.0 \\
\%\end{array}$ & 38 & $76.0 \%$ & 43.03 & $<0.001 * *$ \\
\hline Times per day for catheter cleaning & 21 & $42.0 \%$ & 46 & $92.0 \%$ & 44 & $\begin{array}{c}88.0 \\
\%\end{array}$ & 44 & $88.0 \%$ & 48.48 & $<0.001 * *$ \\
\hline Hard clean around urinary catheter & 3 & $6.0 \%$ & 46 & $92.0 \%$ & 43 & $\begin{array}{c}86.0 \\
\%\end{array}$ & 40 & $80.0 \%$ & 108.55 & $<0.001 * *$ \\
\hline Routine bladder irrigation & 6 & $12.0 \%$ & 43 & $86.0 \%$ & 36 & $\begin{array}{c}72.0 \\
\%\end{array}$ & 34 & $68.0 \%$ & 66.12 & $<0.001^{* *}$ \\
\hline When UC obstructed --- & 19 & $38.0 \%$ & 47 & $94.0 \%$ & 44 & $\begin{array}{c}88.0 \\
\%\end{array}$ & 43 & $86.0 \%$ & 55.93 & $<0.001 * *$ \\
\hline Betadine in U collection bag & 16 & $32.0 \%$ & 49 & $98.0 \%$ & 45 & $\begin{array}{c}90.0 \\
\%\end{array}$ & 44 & $88.0 \%$ & 77.80 & $<0.001 * *$ \\
\hline Uri bag emptying per day & 27 & $54.0 \%$ & 47 & $94.0 \%$ & 46 & $\begin{array}{c}92.0 \\
\%\end{array}$ & 44 & $88.0 \%$ & 36.04 & $<0.001^{* *}$ \\
\hline Nursing care to prevent infection & 11 & $22.0 \%$ & 43 & $86.0 \%$ & 35 & $\begin{array}{c}70.0 \\
\%\end{array}$ & 34 & $68.0 \%$ & 48.04 & $<0.001 * *$ \\
\hline When U bag infected & 16 & $32.0 \%$ & 47 & $94.0 \%$ & 42 & $\begin{array}{c}84.0 \\
\%\end{array}$ & 40 & $80.0 \%$ & 57.45 & $<0.001 * *$ \\
\hline When to sample for urine culture & 29 & $58.0 \%$ & 49 & $98.0 \%$ & 47 & $\begin{array}{c}94.0 \\
\%\end{array}$ & 46 & $92.0 \%$ & 41.42 & $<0.001 * *$ \\
\hline Sterilization of test opening & 21 & $42.0 \%$ & 48 & $96.0 \%$ & 46 & $\begin{array}{c}92.0 \\
\%\end{array}$ & 44 & $88.0 \%$ & 58.50 & $<0.001^{* *}$ \\
\hline Used gloves at sampling & 8 & $16.0 \%$ & 47 & $94.0 \%$ & 43 & $\begin{array}{c}86.0 \\
\%\end{array}$ & 44 & $88.0 \%$ & 98.78 & $<0.001 * *$ \\
\hline before removing urinary catheter & 46 & $88.0 \%$ & 50 & $\begin{array}{c}100.0 \\
\%\end{array}$ & 50 & $\begin{array}{c}100.0 \\
\%\end{array}$ & 50 & $98.0 \%$ & 12.24 & $0.007^{*}$ \\
\hline $\begin{array}{l}\text { Patients ability to urinate after UC } \\
\text { removal }\end{array}$ & 48 & $96.0 \%$ & 50 & $\begin{array}{c}100.0 \\
\%\end{array}$ & 50 & $\begin{array}{c}100.0 \\
\%\end{array}$ & 49 & $98.0 \%$ & 3.72 & $0.27(\mathrm{NS})$ \\
\hline Total & 1 & $2.0 \%$ & 47 & $94.0 \%$ & 42 & $\begin{array}{c}84.0 \\
\%\end{array}$ & 36 & $72.0 \%$ & 111.62 & $<0.001 * *$ \\
\hline
\end{tabular}


Table (4): Percentage distribution of the total score of nurses' knowledge regarding urinary catheterization and care of patients undergoing urinary catheterization throughout the protocol intervention $(\mathrm{No}=50)$

\begin{tabular}{|c|c|c|c|c|c|c|c|c|c|c|c|}
\hline & & \multicolumn{2}{|c|}{$\begin{array}{c}\text { Before } \\
\text { interventio } \\
\mathbf{n}\end{array}$} & \multicolumn{2}{|c|}{$\begin{array}{c}\text { Immediatel } \\
\mathbf{y} \\
\text { After }\end{array}$} & \multicolumn{2}{|c|}{$\begin{array}{l}3 \text { Months } \\
\text { After }\end{array}$} & \multicolumn{2}{|c|}{$\begin{array}{l}6 \text { Months } \\
\text { After }\end{array}$} & \multicolumn{2}{|c|}{ Statistics } \\
\hline & & $\mathbf{N}$ & $\%$ & $\mathbf{N}$ & $\%$ & $\mathbf{N}$ & $\%$ & $\mathbf{N}$ & $\%$ & $\overline{X^{2}}$ & P-value \\
\hline \multirow{4}{*}{$\begin{array}{c}\text { Total } \\
\text { knowledge }\end{array}$} & Satisfied & 5 & 10.0 & 4 & 98.0 & 4 & $96.0 \%$ & 47 & $94.0 \%$ & \multirow{4}{*}{146.2} & \multirow{4}{*}{$<0.001 * *$} \\
\hline & & & $\%$ & 9 & $\%$ & 8 & & & & & \\
\hline & Non- & 4 & 90.0 & 1 & $2.0 \%$ & 2 & $4.0 \%$ & 3 & $6.0 \%$ & & \\
\hline & satisfied & 5 & $\%$ & & & & & & & & \\
\hline \multicolumn{2}{|c|}{ Mean \pm SD } & \multicolumn{2}{|c|}{$\begin{array}{c}61.12 \pm 11.0 \\
1\end{array}$} & \multicolumn{2}{|c|}{$95.61 \pm 5.63$} & \multicolumn{2}{|c|}{$91.07 \pm 7.20$} & \multicolumn{2}{|c|}{$89.75 \pm 7.95$} & $\mathrm{~F}=148.1$ & $<0.001 * *$ \\
\hline \multirow{2}{*}{\multicolumn{2}{|c|}{$\begin{array}{c}\text { immediate Post- pre } \\
\text { protocol }\end{array}$}} & \multirow{2}{*}{\multicolumn{10}{|c|}{$\mathrm{Z}=8.78, \mathrm{p}<0.001^{* *}$}} \\
\hline & & & & & & & & & & & \\
\hline \multicolumn{2}{|c|}{ F3-pre protocol } & \multicolumn{10}{|c|}{$\mathrm{Z}=8.57, \mathrm{p}<0.001^{* *}$} \\
\hline \multicolumn{2}{|c|}{ F6- pre protocol } & \multicolumn{10}{|c|}{$\mathrm{Z}=8.36, \mathrm{p}<0.001^{*}$} \\
\hline \multicolumn{2}{|c|}{$\begin{array}{c}\text { F3-immediately post } \\
\text { protocol }\end{array}$} & \multicolumn{10}{|c|}{$\mathrm{Z}=0.58, \mathrm{p}=0.56(\mathrm{NS})$} \\
\hline \multicolumn{2}{|c|}{$\begin{array}{c}\text { F6-immediately Post } \\
\text { protocol }\end{array}$} & \multicolumn{10}{|c|}{$\mathrm{Z}=1.01, \mathrm{p}=0.31(\mathrm{NS})$} \\
\hline \multicolumn{2}{|c|}{ F6-F3 } & \multicolumn{10}{|c|}{$\mathrm{Z}=0.45, \mathrm{p}=0.64(\mathrm{NS})$} \\
\hline
\end{tabular}

Table (5): Relation between mean score of studied nurses' total satisfactory knowledge regarding care of patients undergoing UC throughout the protocol intervention according to their level of education $(\mathrm{No}=50)$

\begin{tabular}{|c|c|c|c|c|c|c|}
\hline & & \multicolumn{3}{|c|}{ Educational level } & \multirow[t]{3}{*}{ F-test } & \multirow[t]{3}{*}{ P-value } \\
\hline & & $\begin{array}{c}\text { Secondary } \\
\text { diploma }\end{array}$ & $\begin{array}{l}\text { Technical } \\
\text { institute }\end{array}$ & Bachelor & & \\
\hline & & Mean \pm SD & Mean \pm SD & Mean \pm SD & & \\
\hline \multirow{4}{*}{$\begin{array}{c}\text { Total } \\
\text { satisfactory } \\
\text { Knowledge } \\
\text { score }\end{array}$} & Pre protocol & $59.65 \pm 10.78$ & $57.06 \pm 7.99$ & $76.28 \pm 5.24$ & 14.38 & $<0.001^{* *}$ \\
\hline & Immediately after protocol & $94.52 \pm 5.79$ & $95.17 \pm 5.98$ & $99.47 \pm 1.47$ & 2.44 & $0.09(\mathrm{NS})$ \\
\hline & 3 months after protocol & $89.91 \pm 6.77$ & $89.19 \pm 6.95$ & $99.19 \pm 1.45$ & 7.81 & $0.001 * *$ \\
\hline & 6 months after protocol & $89.01 \pm 6.88$ & $87.09 \pm 7.81$ & $99.19 \pm 1.45$ & 9.42 & $<0.001 * *$ \\
\hline
\end{tabular}




\section{DISCUSSION :}

Indwelling urinary catheterization is an invasive intervention with potentially serious outcomes that can lead to morbidity and mortality issues in hospitalized patients so nursing professionals must have sound (and where possible evidence-based) knowledge related to the management of an indwelling urinary catheter, complications caused by an indwelling urinary catheter and ways to prevent and manage these complications (Altun and Karakoc, 2010 and Bernard et al., 2012).

Hence nurses must be fully trained, have knowledge of the underlying principles of nursing care to patient undergoing urinary catheterization, be aware of condition and needs of each patient undergoing urinary catheterization , be familiar of the purposes, indications and complications associated with this procedure. This could enable them to assume the responsibilities of care provided for those patients (Altun and Karakoc, 2010 and Bernard et al., 2012). Moreover Drekonja et al., 2010 added that improved nurses' knowledge can be achieved through education, and leads to practices resulting in decreased CAUTI and other catheter-related complications. Therefore, the aim of this study is to evaluate the impact of protocol of care of patients undergoing urinary catheterization on nurses' performance.

In the same line, Kaushal, (2015) emphasize the positive impact of a training program on the knowledge scores hence the healthcare organizations can engage in continuous training programs to regularly maintain and enhance the knowledge of the nurses .Also CDC, (2013) reported that nurses educated in use and management of indwelling urinary catheter can impact the development of CAUTI and serve to reduce CAUTI risks. The CDC guidelines recommend education include proper insertion techniques for IUCs, management and care , appropriate indications, duration and prevention of potential complications with indwelling urinary catheters .

As regard nurses' knowledge about infection control policies during the urinary catheterization throughout the protocol intervention. Present study findings revealed that improvement of nurses' knowledge regarding the infection control policies during the urinary catheterization care, and their total score. These improvements were high statistically significant differences .This finding is supported by Opina and Oducado, (2014) who stated that low level of knowledge and poor practices on infection control 
in the use of urethral catheters. This indicates that nurses need to be educated and trained more on infection control in the use of urethral catheters. The nurses' level of knowledge had a bearing on their practices on infection control in the use of urethral catheters. Also Kaushal, (2015) reported that continuous training program will significantly increase the knowledge levels of the critical care staff and hence improve their infection control practices.

As regard total score of nurses' knowledge regarding care of patient undergoing urinary catheterization throughout the protocol intervention. It revealed that there were statically significant difference in the total score of nurses' knowledge regarding urinary catheter care throughout the protocol intervention between posttest, pre protocol, first follow up and pre, second follow up, and pre protocol. This result was congruent with that of Drekonja et al. (2009) who stated that, a more effective form of teaching with explanation of the underlying concepts is required to improve knowledge and application of best practice technique for the management of an indwelling urinary catheter, as indicated by the significant improvement in post workshop test scores.

In this respect Talaat et al. (2011) also mentioned that hospital accreditation is still not mandatory in Egypt. However, the Ministry of Health in Egypt recently started developing national accreditation bodies as a preliminary step towards international accreditation of Egyptian hospitals. Limited funds to ensure availability of supplies, lack of personnel with the knowledge and expertise in infection control, and in particular hospital epidemiologists, remain a challenge. Use of devices with outdated technology may also be a factor, such as use of urinary catheters without sampling ports and single lumen catheters for bladder irrigation.

As regard level of education of studied nurses the results revealed that; there were high statically significant differences between the knowledge of studied nurses and their educational level throughout the protocol intervention. The results supported by Prasanna and Radhika,(2015) who reported that there is significant association between the level of knowledge of staff nurses regarding catheter care with their selected socio demographic variables like educational qualification, and source of 
information. The results contradicted with Nasser, (2012) who said that no significant association between Nurses`characteristics and knowledge or practice.

\section{CONCLUSION :}

Based on study findings, it can be concluded that: There was statistically significant improvement of nurses' knowledge regarding the infection control policies during the urinary catheterization. There were statistically significant difference in the total score of nurses' knowledge as well as practice regarding urinary catheter care throughout the protocol intervention between posttest, pre protocol first follow up and pre, second follow up, and pre protocol. A high statistical significant improvement in nurse's knowledge were found between the immediate posttest and the pre protocol level, between pre protocol and 3month after and between pre-second follow up.

\section{RECOMMENDATIONS:}

- Providing ongoing in service education for nurses to update their knowledge related to care of patients undergoing urinary catheterization and catheter -associated problems

- Provide equal opportunities for nurses to attend national and international congresses and in-service training programs related to urinary catheter care.

- Guidelines for infection control of catheters should be reviewed as part of the health authority and considered by both the medical and nursing staff for minimizing infection.

\section{REFERENCES:}

Altun, I., Karakoc, A. (2010): The impact of an interactive workshop on the management of urinary catheterization on nurses. Journal of Urologic Nursing; 4(3): 125-132.

American Association of Colleges of Nursing (AACN) (2014): The Impact of Education on Nursing Practice. Available at http://www.aacn.nche.edu/mediarelations/fact-sheets/impact-of-education.internet accessed at November, 2014. 
Bernard MS., Hunter KF.and Moore K N. (2012): A Review of Strategies to Decrease the Duration of Indwelling Urethral Catheters and Potentially Reduce the Incidence of Catheter- Associated Urinary Tract Infections. Society of Urologic Nurses and Associates, UROLOGIC NURSING; Vol (32) (1); p.29-30.

Centers for Disease Control and Prevention (2013): Catheter-Associated Urinary Tract Infection (CAUTI) Event. Available at:

http://www.cdc.gov/nhsn/PDFs/pscManual/7pscCAUTIcurrent.pdf.

Drekonja DM., Kuskowski MA., and Johnson JR. (2010): Internet survey of Foley catheter practices and knowledge among Minnesota nurses. Am J Infect Control; $38(1): 31-7$.

Essomba1 N., Leme L., Esiene A., bong T., Etoundi O., Gweth N., Abologo L. and Bilong B.(2013) : Identification and quantification of bacteria associated with indwelling urinary catheterization, International Journal of Current Microbiology and Applied Science, Vol2(5) pp. 168-177

Fisher J.(2015): Preventing Catheter Associated Urinary Tract Infections: Implementation of a Nurse Driven Catheter Removal Protocol and Education Program, A Project Presented to the Faculty of California State University, Stanislaus, In Partial Fulfillment of the Requirements for the Degree of Master of Science in Nursing,p.1-12.

Fakih MG., Greene MT., Kennedy EH., Meddings JA., Krein SL., Olmsted RN. and Saint S. (2011): Introducing a population-based outcome measure to evaluate the effect of interventions to reduce catheter-associated urinary tract infection. American Journal of Infection Control. 2012; 40(4): 359-64.

Ignatavicicius DD. and Workman ML. (2013): Medical surgical nursing; patient centered collaborative care; Urinary Elimination; 7th edition, Vol two, Elseiver, USA ;P.1061. 
Perry AG. and Portter PA. (2010): Clinical Nursing Skills Technique, urinary elimination, $7^{\text {th }}$ ed., Elsevier Mosby, USA, P.864.

Weber J., Sickbert-Bennett E., Gould V., Brown M., Huslage K., Rutala A.(2011) :Incidence of catheter-associated and non-catheter-associated urinary tract infections in a healthcare system. Infect Control Hosp Epidemiol.; 32(8):822-823.

Lo E., Niccole, L., Coffin, E., Gould C., Maragakis L., Meddings J., Pegues, A., Pettis M., Saint S. and Yokoe S. (2014): Strategies to prevent catheter-associated urinary tract infections in acute care hospitals: 2014 update. Infection Control and Hospital Epidemiology,35(5). doi:10.1086/675718.

Nasser R. (2012): Effect of In-service Education for Nurses on CAUTI. Faculty of Health Sciences Department of Nursing, Beirut University.

Savagea $A$. (2015): Teaching materials for indwelling urinary catheter management: a case study of one library in Tanzania, International Journal of Urological Nursing Vol 9(3), p.156-164

Kaushal G. (2015): Impact of Training on Knowledge, Attitude and Practices Scores of ICU Nurses regarding Standard Precautions of Infection Control in a Super Speciality Hospital of Delhi, Indian Journal of Research, Volume : 4 | Issue : 8.P.282285.

Opina M. and Oducado R. (2014): Infection Control in the Use of Urethral Catheters: Knowledge and Practices of Nurses in a Private Hospital in Iloilo City. Asia Pacific Journal of Education, Arts and Sciences; Vol. 1 (5); P.93-99.

Prasanna K., Radhika M. (2015): Knowledge regarding Catheter care among Staff Nurses. International Journal of Applied Research 2015; 1(8): 182-186

Talaat M., Hafez, S., Saied T., Elfeky R., El-Shoubary W. and Pimentel G. (2010): Surveillance of catheter-associated urinary tract infection in 4 intensive care units at 
Alexandria university hospitals in Egypt . American Journal of Infection Control, Vol 38(3), P.P.222, 228.

\section{تأثير بروتوكول لرعاية المرضى الخاضعين للقسطرة البولية على معلومات الممرضين}

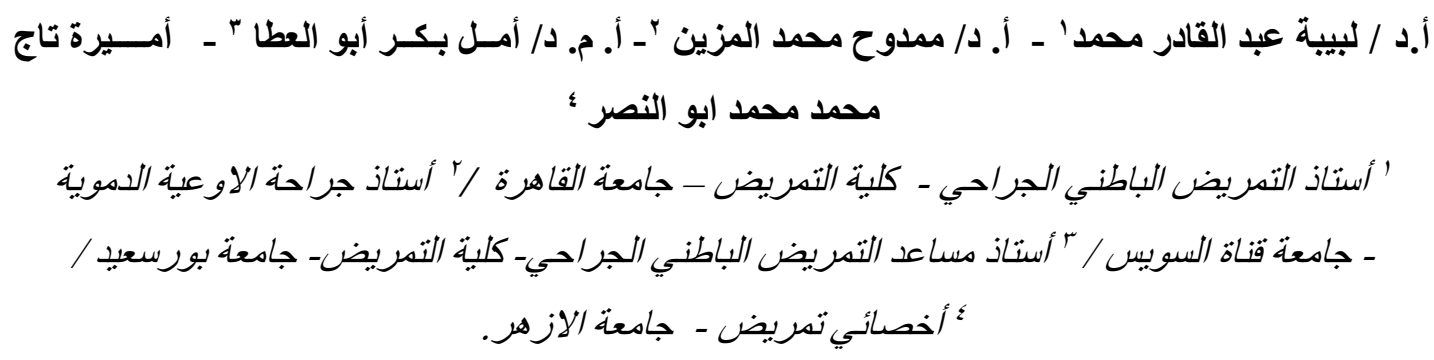

\section{الـخـلاصــة}

تعتبر القسطرة البولية واحدة من أهم و أكثر الاجر اءات العلاجية شيو عاومستخدمة في المستشفيات. وقد يتعـرض المـريض لمخـاطر عديـدة مـن أهمهـا تلـوث مجـرى البـول والتـي يعتبـر أكثر انـواع العـدوى بالمستشفيات حدوثا. وقد أجريت در اسة شبه تجريبية مع تقييم قبلي وبعدى لتقييم تأثير بروتوكول لرعايـة المرضى الخاضعين للقسطرة البوليـة على معلومـات الممرضين الذين يعملون بوحدة الرعايـة المركزة وقسم المسالك البولية وقسم الباطنة الداخلي بمستشفى جامعة الاز هر بدمياط. وقد شملت عينة الدر اسـة على جميع الممرضـات و الممرضين الذين يعملـون بالأمـاكن السـابق ذكر هـا. وقد تـم استخدام استمارة اسـتبيان للبيانـات الثخصـية واستمارة تقيبم معلومـات فيمـا يتعلقت بر عايـة المرضـى الخاضـعين لتركيب القسطرة البوليـة. وقد أسفرت نتائج الدر اسـة عن حدوث تغييـر إيجـابي لمعلومـات الممرضـين بعد تتفيذ بروتوكول حول رعاية المرضى الذين يخضعون للقسطرة البولية وقد أوصت الدر اسـة بتَزويد الممرضـاتِ بالتعليمِ المستمر لتحديث معلوماتهم عن القسطرة البولية ومعرفة المشاكل المصاحبة لها وقد اوصت ايضـا بأهميـة تصـميم بـر امج وبرنوكو لات تعليميـة مسـتمرة لإعطـاء معلومـات عن القسطرة البوليـة لتحسين معلومات الممرضات لتحقيق جودة عالية من الرعاية التمريضية للقسطرة البولية. 